\title{
Valor terapéutico y precio de los nuevos fármacos comercializados en Argentina: ¿valen lo que cuestan?
}

\author{
Therapeutic value and price of the new \\ pharmaceuticals commercialized in Argentina: \\ Are they worth what they cost?
}

Martín Cañás ${ }^{1}$, Héctor Omar Buschiazzo ${ }^{2}$, Martín Alejandro Urtasun ${ }^{3}$

${ }^{1}$ Médico. Magíster en Farmacoepidemiología. Docente, Instituto de Ciencias de la Salud, Universidad Nacional "Arturo Jauretche"; Facultad de Ciencias Médicas, Universidad Nacional de La Plata. Área de Farmacología, Federación Médica de la Provincia de Buenos Aires (FEMEBA), Buenos Aires, Argentina. $\triangle$ iD

${ }^{2}$ Doctor en Medicina. Profesor extraordinario consulto, Facultad de Ciencias Médicas, Universidad Nacional de La Plata. Área de Farmacología, Federación Médica de la Provincia de Buenos Aires (FEMEBA), Buenos Aires, Argentina. $\triangle$ (iD)

${ }^{3}$ Médico. Magíster en Epidemiología, Gestión y Políticas de Salud. Docente, Instituto de Ciencias de la Salud, Universidad Nacional "Arturo Jauretche". Área de Farmacología, Federación Médica de la Provincia de Buenos Aires (FEMEBA), Buenos Aires, Argentina. $\triangle$ iD

\begin{abstract}
RESUMEN En Argentina, los nuevos medicamentos pueden ser autorizados presentando el certificado de aprobación en al menos uno de los 15 países considerados de alta vigilancia sanitaria, sin necesidad de realizar una evaluación propia de eficacia, seguridad o valor terapéutico agregado por el nuevo producto. En este artículo, evaluamos los nuevos medicamentos comercializados en Argentina en el año 2016, utilizando diferentes enfoques: su aprobación por otras agencias reguladoras, demostración de eficacia en ensayos clínicos aleatorizados, tipo de desenlaces estudiados, calificación del valor terapéutico agregado por medio de dos escalas reconocidas y el precio de venta al público. Se concluye que, como reflejo de lo que ocurre en los países desarrollados, los nuevos medicamentos ingresan con precios exorbitantes, pero la mayoría no representa un avance terapéutico significativo. El resultado es un aumento de riesgos para los pacientes y una sobrecarga para los sistemas de financiación públicos y privados.
\end{abstract}

PALABRAS CLAVES Aprobación de Drogas; Evaluación de Medicamentos; Costos de los Medicamentos; Argentina.

\begin{abstract}
In Argentina, new drugs can be authorized by presenting the drug's certificate of approval in at least one of 15 countries considered to have rigorous health surveillance, without needing to carry out a local evaluation of the efficacy, safety or added therapeutic value of the new product. In this article, we evaluate the new drugs commercialized in Argentina in 2016 using different approaches: their approval by other regulatory agencies, the demonstration of their efficacy in randomized clinical trials, types of outcomes studied, rating of their added therapeutic value using two widely recognized scales, and their sale price to the public. It is concluded that, as a reflection of what occurs in developed countries, new drugs enter the market at exorbitant prices, but the majority do not represent a significant therapeutic advancements. The result is increased risks to patients and an overburdening of the public and private funding systems.
\end{abstract}

KEY WORDS Drug Approval; Drug Evaluation; Drug Costs; Argentina. 


\section{INTRODUCCIÓN}

Cada año se incorporan al mercado un sinnúmero de nuevos productos farmacéuticos, que incluyen nuevas presentaciones, nuevas combinaciones o nuevos principios activos. Existe la creencia generalizada, incluso entre los profesionales de la salud, de que todos los medicamentos nuevos aportan una innovación terapéutica y mejores resultados de salud, según lo cual sería deseable el uso de estrategias que aceleren y optimicen su acceso a los pacientes.

El proceso de evaluación por parte de las agencias reguladoras se centra, en general, en cada medicamento individual, que debe probar su eficacia y seguridad en ensayos clínicos controlados de fase III, en muchos casos contra placebo. Así, cuando un medicamento ingresa al mercado, poco sabemos de él en términos comparativos con los productos ya existentes.

Desde una perspectiva de salud pública, el valor de un nuevo medicamento radica en su ganancia terapéutica y en los beneficios para la salud de los pacientes y para la sociedad en su conjunto. Diversos estudios que han evaluado la relevancia clínica de los nuevos medicamentos ingresados en el mercado coinciden en mostrar que la mayor parte de ellos no ofrecen un beneficio terapéutico adicional. De acuerdo a la revisión de la autoridad sanitaria de Canadá, solo un 6\% de los 1.147 nuevos fármacos aprobados entre 1990 y 2003 ofrecían una mejora terapéutica sustancial ${ }^{(1)}$. Por su parte, una evaluación del Instituto Nacional de Gestión Sanitaria de EEUU concluyó que poco menos del $15 \%$ de los 1.035 medicamentos aprobados por la Food and Drug Administration (FDA) entre 1989 y 2000 fueron considerados realmente innovadores ${ }^{(2)}$. Analizando los fármacos introducidos en Brasil entre 2003 y 2013 , se encontró que solo el $17,6 \%$ representaban una "innovación terapéutica importante" ${ }^{\prime \prime(3)}$. Las revisiones de 2007 a 2016 de la revista Prescrire sobre 992 nuevos fármacos o nuevas indicaciones califican solo al $23,3 \%(n=231)$ en alguna de las cuatro categorías que describen algún grado de beneficio ${ }^{(4)}$. Ward et al., utilizando un enfoque más amplio de innovación farmacéutica, encuentran que sobre 290 fármacos incorporados en el periodo 2001-2012 al British National Formulary solo un $26 \%$ son muy innovativos y un $19 \%$, moderadamente innovativos $^{(5)}$. En Australia, el 32\% $(n=19)$ de 59 nuevos fármacos aprobados entre 2005 y 2007 fueron evaluados como de valor terapéutico agregado ${ }^{(6)}$. Analizando 122 nuevos medicamentos autorizados por la European Medicines Agency (EMA) entre 1999 y 2005, van Lujin et al. encuentran que solo el $10 \%$ $(\mathrm{n}=13)$ superan a los fármacos existentes por su efecto sobre desenlaces clínicos ${ }^{(7)}$.

Una buena parte de estos medicamentos presuntamente nuevos son, en realidad, reformulaciones de otros cuya patente está por vencer, o bien moléculas similares a otras ya en uso, que poco o nada aportan a las alternativas existentes, por lo que se han denominado medicamentos "me too" o "yo también". En Argentina los medicamentos nuevos, es decir, aquellos que nunca fueron comercializados en el país, tienen dos vías de aprobación, de acuerdo a la aplicación del Decreto $150 / 1992^{(8)}$. La primera, y más frecuente, es que ya estén comercializados en al menos uno de los 15 países de alta vigilancia sanitaria listados en el Anexo I del Decreto (Alemania, Austria, Bélgica, Canadá, Confederación Helvética, Dinamarca, España, EEUU, Francia, Italia, Israel, Japón, Países Bajos, Reino Unido y Suecia), en cuyo caso solo deben presentar ante la agencia reguladora el certificado de aprobación en dichos países. En el otro caso, cuando el fármaco no fue comercializado previamente en Argentina ni en los países de referencia, el fabricante debe facilitar todos los datos de eficacia y seguridad pertinentes, para llevar a cabo una evaluación completa por parte de la Administración Nacional de Medicamentos, Alimentos y Tecnología Médica (ANMAT).

En la práctica, esto significa que para casi todo el mercado argentino de medicamentos la evaluación de eficacia y seguridad fue realizada por otra agencia nacional y aceptada como válida por las autoridades argentinas. 
Aunque no existe pleno acuerdo, ni un estándar internacional, se han desarrollado varios sistemas de clasificación para categorizar el valor de los nuevos medicamentos. Estos pretenden principalmente ayudar a que los profesionales de la salud y el público dispongan de información sobre el valor terapéutico agregado por los nuevos fármacos sobre las opciones previamente disponibles ${ }^{(9,10,11,12)}$.

Un estudio que evaluó si los fármacos comercializados en EEUU fueron registrados, comercializados y vendidos a precios accesibles en los países latinoamericanos en los que fueron testeados, encontró una gran variación de precios entre ellos, siendo Argentina el país donde se encontraron los precios absolutos más altos para muchos de los medicamentos evaluados ${ }^{(13)}$.

El objetivo de este estudio es evaluar el valor terapéutico de los nuevos medicamentos aprobados por la ANMAT durante el año 2016 y estimar el costo mensual del tratamiento.

\section{MÉTODOS}

Identificamos los medicamentos aprobados para su comercialización en Argentina durante el año 2016 por parte de la ANMAT, consultando las altas de especialidades medicinales de venta libre y de venta bajo receta que publica mensualmente en su sitio web ${ }^{(14)}$. Cuando un mismo principio activo tenía dos presentaciones nuevas con diferentes formas farmacéuticas, se las analizó por separado. Se excluyeron del estudio los productos utilizados para diagnóstico, incluyendo los contrastes radiológicos; las soluciones hidroelectrolíticas, nutricionales o empleadas para irrigación; los productos para hemodiálisis; y las vacunas e inmunoglobulinas.

De acuerdo a la composición de cada especialidad medicinal se la clasificó como monofármaco o combinación de fármacos a dosis fija. Se asignó el código correspondiente de la clasificación Anatómica Terapéutica Química (ATC, por su siglas en inglés) de la Organización Mundial de la Salud, según la consignada en el prospecto autorizado por la ANMAT o, en su defecto, utilizando la base de datos del WHO Collaborative Centre for Drug Statistics Methodology, de acuerdo a los instructivos establecidos ${ }^{(15)}$.

Clasificamos cada especialidad medicinal autorizada en 2016 como previamente comercializada en la Argentina, si ya existía al menos un producto con los mismos principios activos y vía de administración en el catálogo farmacéutico comercial del mes de enero de $2016^{(16)}$, y como nueva especialidad medicinal (NEM) en el resto de los casos. Las NEM correspondían a nuevos principios activos, a nuevas combinaciones de principios activos, o a una nueva vía de administración para un principio activo ya existente. Las diferentes presentaciones que tuvieran iguales principios activos y vía de administración se contabilizaron a los efectos del análisis como una sola NEM. Identificamos las indicaciones de cada NEM según el prospecto autorizado por la ANMAT.

Para evaluar el valor terapéutico de las NEM se utilizaron diferentes aproximaciones: la aprobación por otras agencias reguladoras, la demostración de eficacia en ensayos clínicos aleatorizados y el tipo de desenlaces estudiados, la calificación otorgada por la revista Prescrire y la aplicación de la escala de valor terapéutico de Ahlqvist-Rastad et al.

Se constató si las NEM estaban autorizadas por la $\mathrm{FDA}^{(17)}$, registrando la fecha de aprobación, la prioridad asignada ${ }^{(18)}$ y la condición de fármaco para enfermedades "olvidadas" o "huérfanas". La revisión es "prioritaria" para la FDA cuando la evaluación preliminar muestra que se trata de un fármaco para una enfermedad grave que, de ser aprobado, representaría un avance significativo en la seguridad o efectividad comparado con las opciones disponibles, y es "estándar" en el resto de los casos. Se verificó la aprobación por parte de la $\mathrm{EMA}^{(19)}$, que regula la comercialización de nuevos fármacos en la Unión Europea. Cuando las NEM no estaban aprobadas por la FDA ni por la EMA, se indagó si estaban autorizadas por otros países incluidos en el Decreto $150 / 92^{(8)}$ o si se había realizado 
una evaluación propia de la ANMAT. En este último caso, se consultó la disposición correspondiente de la ANMAT para identificar los criterios de evaluación utilizados.

En segunda instancia se identificaron ensayos clínicos aleatorizados que demostrasen la eficacia de las NEM, tomando como base los descriptos en los prospectos autorizados por la FDA y/o la ANMAT o, en su defecto, realizando una búsqueda en PubMed que combinaba el nombre del principio activo con el filtro para tipo de artículo "randomized controlled trial". Se consideró que una NEM tiene eficacia demostrada en cuatro situaciones: 1) cuando al menos un ensayo clínico aleatorizado muestra eficacia para la indicación autorizada por la ANMAT; 2) en las combinaciones de fármacos, si al menos un ensayo clínico aleatorizado muestra eficacia de los componentes individuales coadministrados y se demuestra la bioequivalencia de la combinación; 3) para nuevas formulaciones de un fármaco, cuando hay eficacia demostrada por ensayos clínicos aleatorizados de la formulación previa y se demuestra bioequivalencia de la nueva presentación; y 4) "medicamentos de eficacia obvia", definidos como aquellos que, sin tener ensayos clínicos aleatorizados de eficacia, se consideran de alto valor intrínseco por sus beneficios inmediatos y obvios en estudios no controlados ${ }^{(20)}$. Se consignó también si la eficacia se había demostrado para variables clínicamente relevantes, para variables subrogadas validadas -aquellas para las que hay fuerte evidencia de que su modificación predice un beneficio clínico específico- o solamente para otras variables subrogadas $^{(21,22,23,24,25)}$.

En tercer lugar, se obtuvo la calificación asignada al nuevo fármaco por la revista Prescrire, una publicación francesa independiente que evalúa los nuevos medicamentos autorizados para calificar el grado de progreso terapéutico, tangible para el paciente, que aporta una NEM en una indicación concreta, planteando el balance beneficio/ riesgo del medicamento con relación a las otras alternativas terapéuticas disponibles ${ }^{(10)}$. Cuando el medicamento tiene varias indicaciones, la calificación de Prescrire puede ser diferente para cada una de ellas. En estos casos, se tomó la mejor calificación dentro de las indicaciones autorizadas por la ANMAT.

Por último, sobre la base de toda la información obtenida, dos autores aplicaron en forma independiente la escala de evaluación del valor terapéutico de Ahlqvist-Rastad et al., que pone énfasis en el grado de novedad del fármaco frente a las opciones previamente disponibles $^{(9)}$ (Tabla 1). La concordancia entre observadores se evaluó con el coeficiente kappa de Cohen y las discrepancias fueron resueltas por consenso.

Tabla 1. Sistema de calificación de Ahlqvist-Rastad et al.

\begin{tabular}{|c|c|c|c|}
\hline \multicolumn{2}{|c|}{ Categoría } & \multicolumn{2}{|c|}{ Subcategoría } \\
\hline A & Fármacos para condiciones que no tienen tratamiento actual & A1 & Con beneficio sustancial para los pacientes \\
\hline & & A2 & Con efecto terapéutico modesto \\
\hline \multirow[t]{4}{*}{ B } & \multirow{4}{*}{$\begin{array}{l}\text { Valor terapéutico agregado: el efecto para los pacientes parece } \\
\text { ser mejor que el de las alternativas disponibles }\end{array}$} & B1 & Mayor eficacia \\
\hline & & B2 & Mayor seguridad \\
\hline & & B3 & Dosificación más conveniente \\
\hline & & B4 & Vía de administración más conveniente \\
\hline \multirow[t]{2}{*}{ c } & \multirow[t]{2}{*}{ Valor terapéutico similar } & C1 & Primer fármaco de una clase nueva \\
\hline & & $\mathrm{C} 2$ & Nuevo fármaco de una clase ya existente \\
\hline \multirow[t]{2}{*}{ D } & \multirow[t]{2}{*}{ Valor terapéutico inferior } & D1 & Primer fármaco de una clase nueva \\
\hline & & D2 & Nuevo fármaco de una clase ya existente \\
\hline $\mathrm{E}$ & $\begin{array}{l}\text { Valor terapéutico incierto: evaluación limitada a desenlaces } \\
\text { subrogados }\end{array}$ & & \\
\hline
\end{tabular}


Como ninguna de las clasificaciones utilizadas hasta aquí considera el costo de los fármacos, se decidió incorporar este aspecto registrando el precio de venta al público publicado en el catálogo farmacéutico comercial Kairos en el momento de aparición en el mercado, y expresando su equivalente en dólares estadounidenses para la cotización de ese día. Para los medicamentos de uso crónico se estimó el precio mensual del tratamiento para la dosis diaria definida por el WHO Collaborative Centre for Drug Statistics Methodology ${ }^{(15)}$. Para los casos en que no está establecida la dosis diaria definida, se utilizó la dosis recomendada en el prospecto del medicamento autorizado por ANMAT, y de faltar esta información, la del prospecto de la FDA. Para los medicamentos de uso esporádico, se registró el precio de venta de un envase. Para el dantrolene, que solo se comercializa en envases de uso hospitalario, se calculó el precio del número promedio de ampollas por episodio de hipertermia maligna, según el prospecto autorizado por la ANMAT.

\section{RESULTADOS}

Entre el 01/01/2016 y el 31/12/2016 la ANMAT autorizó 825 especialidades medicinales, de las que el $10 \%(n=82)$ correspondían a una NEM. En 79 casos se trataba de un nuevo principio activo (o una nueva combinación de principios activos) y en los otros tres casos, de una nueva vía de administración para un principio activo ya existente en el mercado.

Excluimos del análisis los productos para diagnóstico $(n=2)$ y las soluciones para irrigación $(n=1)$. Al contabilizar como un solo medicamento las diferentes presentaciones de los nuevos fármacos (con iguales principios activos y vía de administración) la lista se redujo a 45 NEM incorporadas al mercado argentino en el año 2016, las que se presentan, junto con sus indicaciones autorizadas, según el orden de la clasificación ATC (Tabla 2). La condición de expendio de todas las NEM autorizadas fue de venta bajo receta.

Tabla 2. Nuevas especialidades medicinales autorizadas en Argentina en 2016 y sus indicaciones, ordenadas según los capítulos de la Clasificación Anatómica Terapéutica Química (ATC) de la Organización Mundial de la Salud.

\begin{tabular}{|c|c|c|}
\hline Nueva especialidad medicinal & Indicaciones autorizadas por la ANMAT & Código ATC \\
\hline \multicolumn{3}{|l|}{ A) Tracto alimentario y metabolismo } \\
\hline Colina salicilato + Benzocaína & $\begin{array}{l}\text { Alivio de la inflamación y dolor provocado por ulceraciones, heridas e irritaciones del revestimiento } \\
\text { de la boca. }\end{array}$ & A01AD11 \\
\hline Metformina clorhidrato + Dapagliflozina & $\begin{array}{l}\text { Adyuvante de la dieta y del ejercicio para mejorar el control glucémico en adultos con diabetes } \\
\text { mellitus tipo } 2 \text { cuando el tratamiento tanto con dapagliflozina como con metformina es apropiado. }\end{array}$ & A10BD15 \\
\hline Nitisinona & Tirosinemia hereditaria tipo 1 & A16AX04 \\
\hline \multicolumn{3}{|l|}{ B) Sangre y órganos formadores de sangre } \\
\hline Epoprostenol & Hipertensión arterial pulmonar. Anticoagulación en diálisis renal cuando no se puede usar heparina. & B01AC09 \\
\hline Eltrombopag & $\begin{array}{l}\text { Trombocitopenia en adultos y niños mayores de } 6 \text { años con púrpura trombocitopénica idiopática } \\
\text { crónica con respuesta insuficiente a corticosteroides, inmunoglobulina o esplenectomía / } \\
\text { Trombocitopenia en pacientes con hepatitis C crónica para permitir el tratamiento a base } \\
\text { del interferón / Anemia aplásica severa que ha tenido una respuesta insuficiente a la terapia } \\
\text { inmunosupresora. }\end{array}$ & B02BX05 \\
\hline \multicolumn{3}{|l|}{ C) Sistema cardiovascular } \\
\hline Ambrisentan & Hipertensión arterial pulmonar clase funcional II y III de la OMS. & $\mathrm{CO2KX02}$ \\
\hline Riociguat & $\begin{array}{l}\text { Hipertensión pulmonar tromboembólica crónica e Hipertensión arterial pulmonar, clase funcional } \\
\text { II o III. }\end{array}$ & C02KX05 \\
\hline Sacubitrilo + Valsartán & Insuficiencia cardíaca crónica clase II-IV con fracción de eyección disminuida. & C09DX04 \\
\hline Rosuvastatina + Ezetimibe & Hipercolesterolemia primaria (heterocigota familiar y no familiar). & C10BA06 \\
\hline
\end{tabular}


Tabla 2. Continuación.

D) Medicamentos dermatológicos

Concentrado de enzimas proteolíticas enriquecidas con bromelaína

Aciclovir + Hidrocortisona

Herpes labial recurrente.

D03BA03

Hidroquinona

Blanqueamiento gradual de la piel hiperpigmentada.

D11AX11

G) Medicamentos urológicos

Ácido hialurónico (uso intravesical)

Tratamiento sintomático de cistitis intersticial, cistitis causadas por infecciones recurrentes, urolitiasis, retención de la orina, neoplasia y cistitis inducida por radiación.

H) Preparados hormonales sistémicos, excluyendo hormonas sexuales

Carbetocina

Prevención de atonía uterina y hemorragia postparto después de una cesárea por elección con

H01BB03 anestesia epidural o raquídea.

J) Medicamentos antiinfecciosos generales de uso sistémico

Posaconazol (comprimidos de liberacion modificada)

Posaconazol (parenteral)

Emtricitabina +Tenofovir disoproxil fumarato + Rilpivirina

Abacavir + Lamivudina + Dolutegravir

Atazanavir + Cobicistat

Ombitasvir + Paritaprevir + Ritonavir + Dasabuvir
Prevención de infecciones micóticas invasivas causadas por Aspergillus y Candida en pacientes inmunocomprometidos. Tratamiento de infecciones por Aspergillus que no mejoran con anfotericina B o itraconazol, o cuando estos medicamentos deben suspenderse.

Tratamiento de infecciones por Aspergillus, Fusarium, cromoblastomicosis, micetoma, Coccidiodes, que no han respondido a otros tratamientos. También prevención de micosis en pacientes de alto riesgo (leucemia mieloide aguda, sindrome mielodisplásicos, trasplante autólogo de médula ósea).

Régimen completo para el tratamiento de la infección por VIH-1 en pacientes adultos sin antecedentes de tratamiento antirretroviral y con ARN del VIH-1 menor o igual a 100.000 copias $/ \mathrm{ml}$ al comienzo del tratamiento y en ciertos pacientes adultos con supresión virológica definida como ARN del VIH-1 $<50$ copias/ml y con un régimen antirretroviral estable al comienzo del tratamiento, para sustituir su régimen de tratamiento antirretroviral actual.

Adultos y adolescentes mayores de 12 años de edad infectados por el VIH y que pesen al menos $40 \mathrm{~kg}$. Antes de iniciar el tratamiento con medicamentos que contengan abacavir, se debe llevar a cabo una prueba de detección del alelo HLA-B*5701 en todos los pacientes infectados por el VIH, independientemente del origen racial. No se debe emplear abacavir en pacientes portadores del alelo HLA-B*5701.

Indicado en combinación con otros agentes antirretrovirales para el tratamiento de la infección por el virus de inmunodeficiencia humana (VIH-I) en adultos.

Hepatitis C Genotipo 1, incluyendo aquellos con cirrosis compensada
J02AC04

$\mathrm{J02ACO4}$

J05AR08

J05AR13

J05AR15

J05AX66

L) Agentes antineoplásicos e inmunomoduladores

Brentuximab vedotina

Linfoma Hodgkin en recaída o refractario / Linfoma anaplásico de células grandes sistémico en recaída o refractario.

L01XC12

L01XC15 dosis plena no es adecuado.

Nivolumab

Melanoma irresecable o metastásico / Cáncer de pulmón metastásico de células no pequeñas / Cáncer de células renales / Linfoma de Hodgkin.

Melanoma irresecable o metastásico.

L01XC17

L01XC18

L01XE07

(ErbB2): en combinación con capecitabina para ermedad avanzada o metastásica que ha progresado tras haber recibido tratamiento previo que incluya antraciclinas y taxanos, y tratamiento con trastuzumab en enfermedad metastásica / En combinación con trastuzumab en pacientes con enfermedad metastásica y receptor hormonal negativo que ha progresado a terapia(s) previa(s) con trastuzumab en combinación con quimioterapia / En combinación con un inhibidor de aromatasa en mujeres posmenopáusicas con enfermedad metastásica y receptores hormonales positivos, para las cuales la quimioterapia no es adecuada.

Trametinib

Melanoma con mutación en el gen BRAF y que no han recibido un inhibidor BRAF.

L01XE25

Linfoma de células del manto / Leucemia linfocítica crónica / Leucemia linfocítica crónica con deleción del cromosoma $17 \mathrm{p}$.

Fibrosis pulmonar idiopática.

L01XE31

Cáncer de mama avanzado con ER(+) y HER2(-) en post-menopausia, asociado a letrozol, como terapia endocrina inicial para enfermedad metastática.

L01XE33

Palbociclib

Monoterapia de mantenimiento para cáncer de ovario seroso de alto grado recaído con BRCA mutado y sensible a platino, que estén respondiendo a quimioterapia a base de platino. 
Tabla 2. Continuación.

\begin{tabular}{|c|c|c|}
\hline Nueva especialidad medicinal & Indicaciones autorizadas por la ANMAT & Código ATC \\
\hline Venetoclax & Leucemia linfocítica crónica con deleción 17p, que ha recibido al menos una terapia previa. & L01XX52 \\
\hline Mifamurtida & $\begin{array}{l}\text { Osteosarcoma de alto grado resecable no metastásico después de una resección quirúrgica } \\
\text { macroscópicamente completa, en niños, adolescentes y adultos jóvenes. }\end{array}$ & L03AX15 \\
\hline Teriflunomida & Esclerosis múltiple remitente recurrente. & L04AA31 \\
\hline Ustekinumab & Psoriasis en placas moderada a grave / Artritis psoriática activa. & L04AC05 \\
\hline Pomalidomida & $\begin{array}{l}\text { Asociada a dexametasona, en adulto con mieloma múltiple resistente y recidivante, que haya } \\
\text { recibido al menos dos tratamientos previos, incluyendo lenalinomida y bortezomib, y que haya } \\
\text { experimentado progresión de la enfermedad en el último tratamiento. }\end{array}$ & L04AX06 \\
\hline \multicolumn{3}{|l|}{ M) Sistema musculoesquelético } \\
\hline Dantrolene sódico 3 y $1 / 2$ hidrato & Hipertermia maligna & M03CA01 \\
\hline $\begin{array}{l}\text { Ácido hialurónico sal sódica + Lidocaína } \\
\text { clorhidrato }\end{array}$ & $\begin{array}{l}\text { Tratamiento sintomático del dolor y de la función articular en artrosis de rodilla, en la periartritis del } \\
\text { hombro y otras articulaciones sinoviales. }\end{array}$ & M09AX01 \\
\hline \multicolumn{3}{|l|}{ N) Sistema nervioso } \\
\hline Doxepina & Insomnio primario, tratamiento a corto plazo. & N06AA12 \\
\hline \multicolumn{3}{|l|}{ R) Sistema respiratorio } \\
\hline Olodaterol & $\begin{array}{l}\text { Tratamiento de mantenimiento a largo plazo en un régimen de administración diaria en pacientes } \\
\text { con enfermedad pulmonar obstructiva crónica (EPOC). }\end{array}$ & R03AC19 \\
\hline Umeclidinio + Vilanterol & $\begin{array}{l}\text { Tratamiento broncodilatador de mantenimiento para aliviar los síntomas de la EPOC en pacientes } \\
\text { adultos. }\end{array}$ & R03AL03 \\
\hline Olodaterol + Bromuro de tiotropio & $\begin{array}{l}\text { Tratamiento de mantenimiento a largo plazo en un régimen de administración diaria en pacientes } \\
\text { con EPOC. }\end{array}$ & R03AL06 \\
\hline Umeclidinio bromuro & $\begin{array}{l}\text { Tratamiento broncodilatador de mantenimiento para aliviar los síntomas de la EPOC en pacientes } \\
\text { adultos. }\end{array}$ & R03BB07 \\
\hline \multicolumn{3}{|l|}{ S) Órganos de los sentidos } \\
\hline Brimonidina + Brinzolamida & $\begin{array}{l}\text { Glaucoma de ángulo abierto o hipertensión ocular en los que la monoterapia produce una reducción } \\
\text { insuficiente de la presión intraocular. }\end{array}$ & S01EC54 \\
\hline \multicolumn{3}{|l|}{ V) Varios } \\
\hline Citrulina malato & $\begin{array}{l}\text { Astenia, una vez descartado que esta sea secundaria a una enfermedad de base de cualquier causa, } \\
\text { sea física o psiquiátrica. }\end{array}$ & V06DD \\
\hline Dicloruro de radio 223 & $\begin{array}{l}\text { Agente terapéutico emisor de partículas radioactivas alfa indicado para el tratamiento de } \\
\text { pacientes con cáncer de próstata resistente a la castración con metástasis óseas sintomáticas y sin } \\
\text { enfermedad visceral metastásica conocida. }\end{array}$ & V10XX03 \\
\hline
\end{tabular}

Casi un tercio de las NEM $(31 \%, \mathrm{n}=14)$ fueron combinaciones con más de un principio activo. Los fármacos antineoplásicos e inmunomoduladores fueron la clase terapéutica con más novedades $(33 \%, n=15$, incluyendo cinco anticuerpos monoclonales y seis inhibidores de tirosina-kinasas), seguidas por los antiinfecciosos (13\%, $\mathrm{n}=6)$ ) (Figura 1).

\section{Aprobación por otras agencias reguladoras}

La mayoría de las NEM aprobadas por la ANMAT en 2016 habían sido previamente autorizadas por la FDA $(80 \%, \mathrm{n}=36)$ y por la EMA $(78 \%, n=35)$. De las seis NEM que no estaban autorizadas por ninguna de las dos 


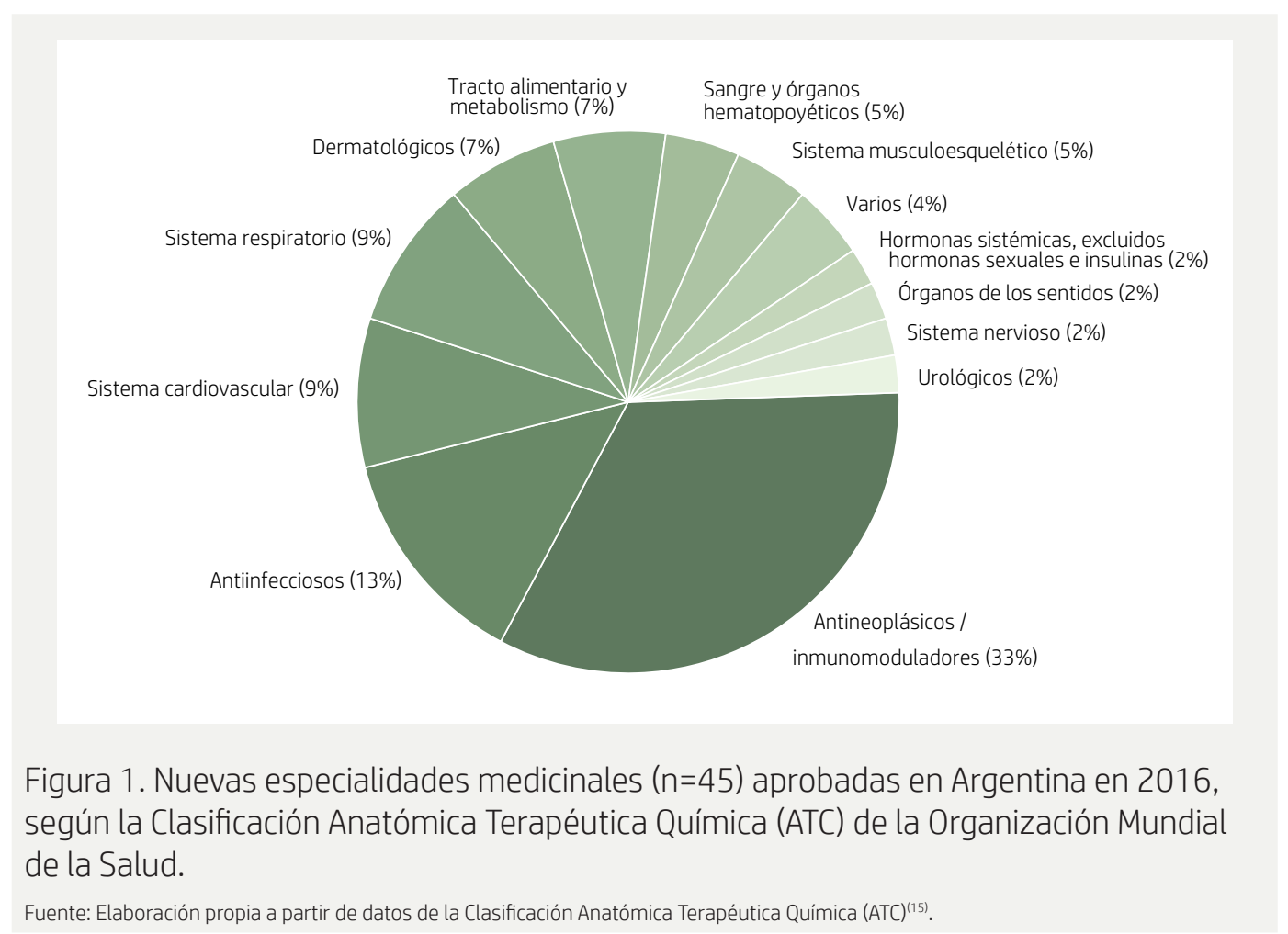

agencias, cuatro tenían aprobación en países del Anexo I del Decreto 150/92 (carbetocina, hidroquinona, rosuvastatina + ezetimibe $y$ citrulina malato) y las 2 restantes fueron evaluadas primariamente por la ANMAT (ácido hialurónico + lidocaína para uso intraarticular y salicilato de colina + benzocaína como tratamiento tópico bucal). De las 36 NEM aprobadas por FDA, $53 \%(n=19)$ calificaron para revisión prioritaria y $42 \%(n=15)$ para enfermedades huérfanas.

\section{Fármacos evaluados primariamente por la ANMAT}

Los criterios identificados en las dos evaluaciones originales de la ANMAT fueron diversos. En el caso del producto con salicilato de colina y benzocaína para tratamiento tópico bucal, la aprobación se basó en la eficacia y seguridad de los componentes por separado, sin referencia a estudios que evalúen la combinación a dosis fija ${ }^{(26)}$. En cuanto a la asociación de ácido hialurónico con lidocaína para uso intraarticular, se encuadró como una nueva presentación del ácido hialurónico previamente aprobado, y no como una nueva especialidad medicinal ${ }^{(27)}$.

\section{Eficacia comprobada en ensayos controlados aleatorizados}

Para el $62 \%$ de las NEM $(n=28)$ se encontraron ensayos clínicos aleatorizados que mostraban eficacia; otro $20 \%(n=9)$ eran nuevas combinaciones o nuevas formulaciones de fármacos que ya tenían ensayos clínicos aleatorizados de eficacia, en los que solo se constató para su autorización la bioequivalencia del nuevo preparado (Tabla 3).

Del $18 \%$ de las NEM $(n=8)$ sin ensayos clínicos aleatorizados de eficacia, un $4 \%$ $(\mathrm{n}=2)$ pueden categorizarse como fármacos "de eficacia obvia", a saber, el dantrolene para la hipertermia maligna y la nitisinona en la tirosinemia hereditaria. El 13\% $(n=6)$ restante no tenían ensayos clínicos aleatorizados de eficacia; dos de ellos, antineoplásicos, fueron evaluados en ensayos no controlados. 
Tabla 3. Evaluación de las nuevas especialidades medicinales autorizadas en Argentina, en 2016, según existencia de ensayos clínicos aleatorizados de eficacia, tipo de desenlace con beneficio demostrado, calificación de la revista Prescrire y la escala de Ahlqvist-Rastad et al.

\begin{tabular}{|c|c|c|c|c|c|}
\hline Código ATC & Nueva especialidad medicinal & $\begin{array}{c}\text { Ensayos clínicos } \\
\text { aleatorizados de } \\
\text { eficacia }\end{array}$ & $\begin{array}{c}\text { Tipo de desenlace } \\
\text { con beneficio } \\
\text { demostrado }\end{array}$ & $\begin{array}{l}\text { Calificación } \\
\text { Prescrire }\end{array}$ & $\begin{array}{l}\text { Escala Ahlquist- } \\
\text { Rastad et al. }\end{array}$ \\
\hline \multicolumn{6}{|c|}{ A) Tracto alimentario y metabolismo } \\
\hline A01AD11 & Colina salicilato + Benzocaina & No & No & Inaceptable & $E$ \\
\hline A10BD15 & Metformina clorhidrato + Dapagliflozina & Sí-CDF & Subrogado-NV & Inaceptable & $\mathrm{D} 2$ \\
\hline A16AX04 & Nitisinona & Eficacia obvia & Relevante & Bravo & A1 \\
\hline \multicolumn{6}{|c|}{ B) Sangre y órganos formadores de sangre } \\
\hline B01AC09 & Epoprostenol & Sí & Relevante & Ofrece una ventaja & $\mathrm{C} 1$ \\
\hline B02BX05 & Eltrombopag & Sí & Relevante & Nada nuevo & $\mathrm{C} 1$ \\
\hline
\end{tabular}

\section{C) Sistema cardiovascular}

$\begin{array}{llcc}\text { C02KX02 } & \text { Ambrisentan } & \text { Sí } & \text { Subrogado-NV } \\ \text { C02KX05 } & \text { Riociguat } & \text { Sí } & \text { Subrogado-NV } \\ \text { C09DX04 } & \text { Sacubitrilo + Valsartán } & \text { Sí } & \text { Resiblemente útil } \\ \text { C10BA06 } & \text { Rosuvastatina + Ezetimibe } & \text { Sí-CDF } & \text { Subrogado-V }\end{array}$

D) Medicamentos dermatológicos

\begin{tabular}{|c|c|c|c|c|c|}
\hline D03BA03 & $\begin{array}{l}\text { Concentrado de enzimas proteoliticas enriquecidas con } \\
\text { bromelaina }\end{array}$ & Sí & Relevante & No evaluado & B1 \\
\hline D06BB53 & Aciclovir + Hidrocortisona & Sí & Relevante & Inaceptable & $\mathrm{C} 2$ \\
\hline D11AX11 & Hidroquinona & Sí & Relevante & Inaceptable & D2 \\
\hline \multicolumn{6}{|c|}{ G) Medicamentos urológicos } \\
\hline G04BX & Ácido hialurónico (uso intravesical) & No & No & No evaluado & $\mathrm{E}$ \\
\hline \multicolumn{6}{|c|}{ H) Preparados hormonales sistémicos, excluyendo hormonas sexuales } \\
\hline Н01ВВ03 & Carbetocina & Sí & Relevante & Nada nuevo & $\mathrm{C} 2$ \\
\hline \multicolumn{6}{|c|}{ J) Medicamentos antiinfecciosos generales de uso sistémico } \\
\hline J02AC04 & Posaconazol (comprimidos de liberacion modificada) & Sí-NF & Relevante & Posiblemente útil & $\mathrm{C} 2$ \\
\hline J02AC04 & Posaconazol (parenteral) & Sí-NF & Relevante & Posiblemente útil & B4 \\
\hline J05AR08 & $\begin{array}{l}\text { Emtricitabina + Tenofovir disoproxil fumarato + } \\
\text { Rilpivirina }\end{array}$ & Sí-CDF & Subrogado-V & Nada nuevo & $\mathrm{C} 2$ \\
\hline J05AR13 & Abacavir + Lamivudina + Dolutegravir & Sí-CDF & Subrogado-V & Nada nuevo & $\mathrm{C} 2$ \\
\hline J05AR15 & Atazanavir + Cobicistat & Sí-CDF & Subrogado-V & Nada nuevo & $\mathrm{C} 2$ \\
\hline J05AX66 & Ombitasvir + Paritaprevir + Ritonavir + Dasabuvir & Sí-CDF & Subrogado-V & Nada nuevo & $\mathrm{C} 2$ \\
\hline
\end{tabular}

\begin{tabular}{|c|c|c|c|c|c|}
\hline \multicolumn{6}{|c|}{ L) Agentes antineoplásicos e inmunomoduladores } \\
\hline L01XC12 & Brentuximab vedotina & Sí & Relevante & Juicio reservado & C1 \\
\hline L01XC15 & Obinutuzumab & Sí & Relevante & Nada nuevo & $\mathrm{C} 2$ \\
\hline L01XC17 & Nivolumab & Sí & Relevante & $\begin{array}{l}\text { Un verdadero } \\
\text { avance }\end{array}$ & B1 \\
\hline L01XC18 & Pembrolizumab & Sí & Relevante & Nada nuevo & $\mathrm{C} 2$ \\
\hline L01XE07 & Lapatinib & Sí & Subrogado-NV & Posiblemente útil & C1 \\
\hline
\end{tabular}


Tabla 3. Continuación.

\begin{tabular}{|c|c|c|c|c|c|}
\hline Código ATC & Nueva especialidad medicinal & $\begin{array}{l}\text { Ensayos clínicos } \\
\text { aleatorizados de } \\
\text { eficacia }\end{array}$ & $\begin{array}{l}\text { Tipo de desenlace } \\
\text { con beneficio } \\
\text { demostrado }\end{array}$ & $\begin{array}{l}\text { Calificación } \\
\text { Prescrire }\end{array}$ & $\begin{array}{l}\text { Escala Ahlquist- } \\
\text { Rastad et al. }\end{array}$ \\
\hline L01XE25 & Trametinib & Sí & Relevante & Ofrece una ventaja & B1 \\
\hline L01XE27 & Ibrutinib & Sí & Relevante & Juicio reservado & B1 \\
\hline L01XE31 & Nintedanib & Sí & Subrogado-NV & Inaceptable & E \\
\hline L01XE33 & Palbociclib & Si & Subrogado-NV & Inaceptable & $\mathrm{E}$ \\
\hline L01XX46 & Olaparib & No-Última línea & Subrogado-NV & Inaceptable & E \\
\hline L01XX52 & Venetoclax & No-Última línea & Subrogado-NV & No evaluado & C1 \\
\hline L03AX15 & Mifamurtida & Si & Subrogado-NV & Inaceptable & E \\
\hline L04AA31 & Teriflunomida & Sí & Subrogado-NV & Inaceptable & D2 \\
\hline L04AC05 & Ustekinumab & Sí & Relevante & Nada nuevo & C2 \\
\hline L04AX06 & Pomalidomida & Sí & Relevante & Posiblemente útil & B1 \\
\hline \multicolumn{6}{|c|}{ M) Sistema musculoesquelético } \\
\hline M03СА01 & Dantrolene sódico 3 y $1 / 2$ hidrato & Eficacia obvia & Relevante & De elección* & $\mathrm{A} 1$ \\
\hline M09AX01 & Ácido hialurónico sal sódica + Lidocaína clorhidrato & No & No & Inaceptable & E \\
\hline \multicolumn{6}{|c|}{ N) Sistema nervioso } \\
\hline N06AA12 & Doxepina & Sí & Subrogado-NV & No evaluado & D2 \\
\hline \multicolumn{6}{|c|}{ R) Sistema respiratorio } \\
\hline R03AC19 & Olodaterol & Sí & Subrogado-V & Nada nuevo & $\mathrm{C} 2$ \\
\hline R03AL03 & Umeclidinio + Vilanterol & Sí & Subrogado-V & Nada nuevo & $\mathrm{C} 2$ \\
\hline R03AL06 & Olodaterol + Bromuro de tiotropio & Sí & Subrogado-V & Nada nuevo & $\mathrm{C2}$ \\
\hline R03BB07 & Umeclidinio bromuro & Si & Subrogado-V & Nada nuevo & $\mathrm{C} 2$ \\
\hline \multicolumn{6}{|c|}{ S) Órganos de los sentidos } \\
\hline S01EC54 & Brimonidina + Brinzolamida & Sí-CDF & Subrogado-V & Nada nuevo & $\mathrm{C2}$ \\
\hline \multicolumn{6}{|l|}{ V) Varios } \\
\hline V06DD & Citrulina malato & No & No & Nada nuevo & E \\
\hline V10XX03 & Dicloruro de Radio 223 & Sí & Relevante & No evaluado & B1 \\
\hline
\end{tabular}

El segundo aspecto a evaluar fue si se había comprobado eficacia para desenlaces clínicamente relevantes o para desenlaces subrogados (validados o no validados). EI $44 \%(n=20)$ de las NEM habían demostrado eficacia en desenlaces relevantes; otro $22 \%$ $(n=10)$ fueron eficaces sobre variables subrogadas validadas, a saber, Volumen Espiratorio Forzado en el primer segundo (VEF1) en
Enfermedad Pulmonar Obstructiva Crónica $(\mathrm{EPOC})^{(28,29)}$, carga viral indetectable en fármacos para $\mathrm{VIH}^{(22)}$, respuesta viral sostenida para hepatitis $\mathrm{C}^{(30,31)}$, descenso de las lipoproteínas de baja densidad (LDL) para hipolipemiantes ${ }^{(22)}$ y presión intraocular en el tratamiento del glaucoma ${ }^{(23)}$. Entre las restantes NEM autorizadas, un $24 \%(n=11)$ demostraron eficacia solo en variables subrogadas 
Tabla 4. Existencia de ensayos clínicos aleatorizados y tipo de desenlaces evaluados para las nuevas especialidades medicinales aprobadas en Argentina en 2016.

\begin{tabular}{|c|c|c|c|c|c|}
\hline \multirow[t]{3}{*}{ ¿Hay ECA de eficacia? } & \multicolumn{3}{|c|}{ Hay pruebas de eficacia } & \multirow{3}{*}{$\begin{array}{l}\text { No hay } \\
\text { pruebas de } \\
\text { eficacia }\end{array}$} & \multirow[t]{3}{*}{ Total } \\
\hline & \multirow[t]{2}{*}{$\begin{array}{l}\text { En desenlaces } \\
\text { relevantes }\end{array}$} & \multicolumn{2}{|c|}{$\begin{array}{l}\text { En desenlaces } \\
\text { subrogados }\end{array}$} & & \\
\hline & & Validados & No validados & & \\
\hline Sí & 16 & 4 & 8 & - & 28 \\
\hline Sí - Combinación a dosis fija & - & 6 & 1 & - & 7 \\
\hline Sí - Nueva formulación & 2 & - & - & - & 2 \\
\hline Fármacos de eficacia obvia & 2 & - & - & - & 2 \\
\hline No - Oncológicos de última línea & - & - & 2 & - & 2 \\
\hline No & - & - & - & 4 & 4 \\
\hline Total & 20 & 10 & 11 & 4 & 45 \\
\hline
\end{tabular}

no validadas y un $9 \%(\mathrm{n}=4)$ no tenían pruebas de eficacia. Estos resultados se detallan en la Tabla 3 y se resumen en la Tabla 4.

\section{Clasificación asignada por la revista Prescrire}

De las 45 NEM autorizadas en 2016 hubo 39 evaluadas en la revista Prescrire. Más del
$70 \%$ de ellas no presentaban ventajas: la categoría más numerosa fue la de los fármacos que no aportan nada nuevo $(41 \%, \mathrm{n}=16)$, con otro $26 \%(n=10)$ calificados como inaceptables y un $5 \%(n=2)$ con juicio reservado. Solo el $28 \%$ de las NEM evaluadas por Prescrire $(\mathrm{n}=11)$ quedaron clasificadas en las categorías que representan un avance, aunque sea mínimo, sobre las opciones terapéuticas previas (Tabla 5).

Tabla 5. Evaluación de la revista Prescrire sobre las nuevas especialidades medicinales ( $n=39)$ aprobadas en Argentina en 2016.

\begin{tabular}{|c|c|c|c|}
\hline Categoría & Concepto & Cantidad & $\%$ \\
\hline Bravo & $\begin{array}{l}\text { Un avance terapéutico importante en un área sin tratamiento } \\
\text { previo disponible }\end{array}$ & 1 & 2,6 \\
\hline De elección ${ }^{a}$ & & 1 & 2,6 \\
\hline Un verdadero avance & Un avance terapéutico importante, con ciertas limitaciones & 1 & 2,6 \\
\hline Ofrece una ventaja & $\begin{array}{l}\text { El producto tiene algún valor, pero no cambia fundamentalmente } \\
\text { la práctica terapéutica actual }\end{array}$ & 2 & 5,1 \\
\hline Posiblemente útil & $\begin{array}{l}\text { Tiene mínimo valor adicional, y no debería cambiar la práctica } \\
\text { prescriptiva excepto en circunstancias poco frecuentes }\end{array}$ & 6 & 15,4 \\
\hline Nada nuevo & $\begin{array}{l}\text { El producto es una sustancia nueva pero no hay evidencia de que } \\
\text { tenga más valor clínico que otras sustancias del mismo grupo }\end{array}$ & 16 & 41,0 \\
\hline Inaceptable & $\begin{array}{l}\text { Producto sin beneficio evidente, pero con desventajas reales o } \\
\text { potenciales }\end{array}$ & 10 & 25,6 \\
\hline Juicio reservado & $\begin{array}{l}\text { Se pospone la calificación hasta que haya datos mejores y sea } \\
\text { posible una evaluación más completa }\end{array}$ & 2 & 5,1 \\
\hline
\end{tabular}




\section{Evaluación del valor terapéutico}

Hubo buena concordancia interobservador en la asignación del valor terapéutico de las NEM $(\kappa=0,90)$. La evaluación, según la escala de Ahlqvist-Rastad et al., muestra que la mayoría de las NEM ( $51 \%, \mathrm{n}=23)$ no tienen valor terapéutico agregado (categoría C), otro $9 \%$ $(n=4)$ se consideran inferiores a las alternativas disponibles (categoría D) y un $18 \%(\mathrm{n}=8)$ tiene valor terapéutico incierto (E). Solamente un $22 \%(n=10)$ de las NEM representan algún grado de valor terapéutico agregado (categorías A y B) (Figura 2 y Tabla 3).

\section{Precio de venta al público de las nuevas especialidades medicinales}

De las 45 NEM autorizadas, hubo cinco de uso esporádico y 40 de uso crónico. En 20 de estas últimas se calculó el precio mensual de la dosis diaria definida, en 17 la dosis del prospecto ANMAT y en uno la del prospecto FDA. Hubo dos productos (hidroquinona, nitisinona) para los que no fue posible obtener el precio de venta.
El precio del tratamiento con los nuevos medicamentos de uso crónico muestra una gran dispersión, desde 13 a $\$ 56.516$ dólares mensuales. El precio de la mayoría de los fármacos fue elevado, con un promedio mensual de $\$ 7.974$ dólares y mediana de $\$ 5.849$ dólares (intervalo intercuartílico: $\mathrm{Q} 1=\$ 172 ; \mathrm{Q} 3=\$ 14.284)$. Para las NEM que agregan valor terapéutico, el precio fue más elevado, con promedio de $\$ 13.800$ dólares (mediana $=\$ 13.821$; intervalo intercuartílico: $\mathrm{Q} 1=\$ 5.021 ; \mathrm{Q} 3=\$ 13.325)$.

Al agrupar los fármacos según su indicación principal se observa cómo se concentran en diferentes franjas de precio de acuerdo a la patología tratada (Figura 3). Puede observarse que el precio mensual del tratamiento de los 14 fármacos de uso oncológico va de $\$ 3.000$ a $\$ 56.000$ dólares, con un promedio de $\$ 17.700$. En contraste con este dato, solo cinco de ellos tuvieron valor terapéutico agregado, otros cinco fueron de valor similar al de los fármacos existentes, tres de valor terapéutico incierto y uno se consideró inferior a las alternativas disponibles.

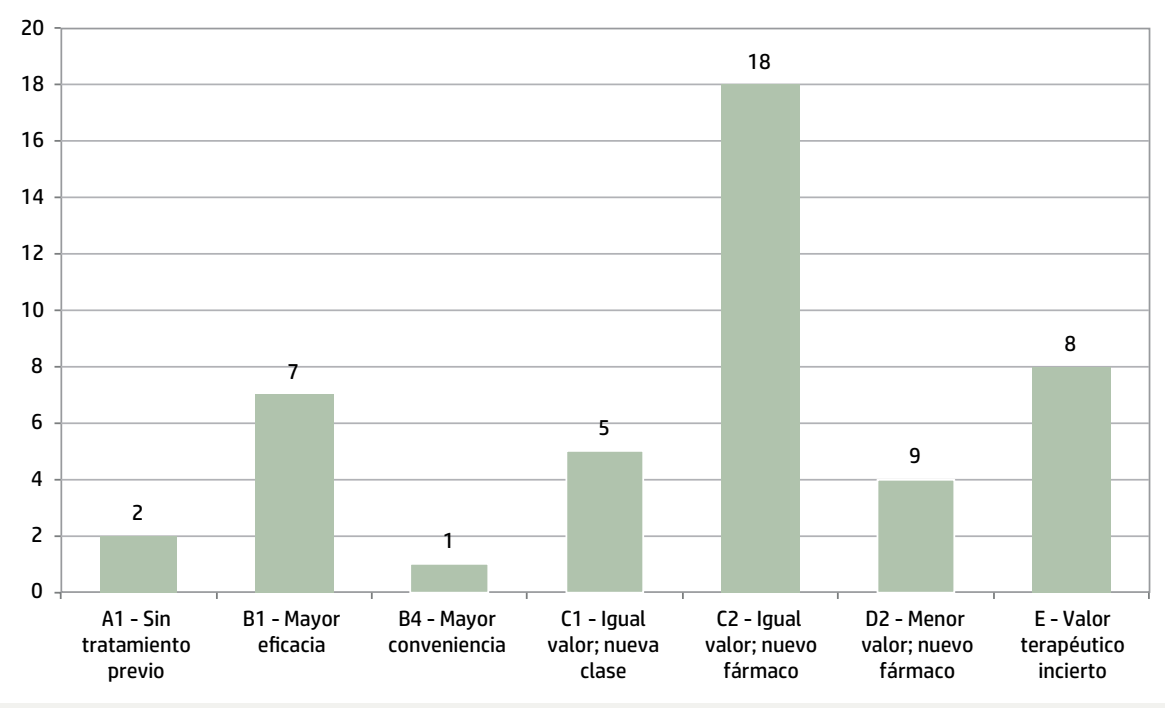

Figura 2. Valor terapéutico de las nuevas especialidades medicinales $(n=45)$ aprobadas en Argentina en 2016, según clasificación de Ahlqvist-Rastad et al. 


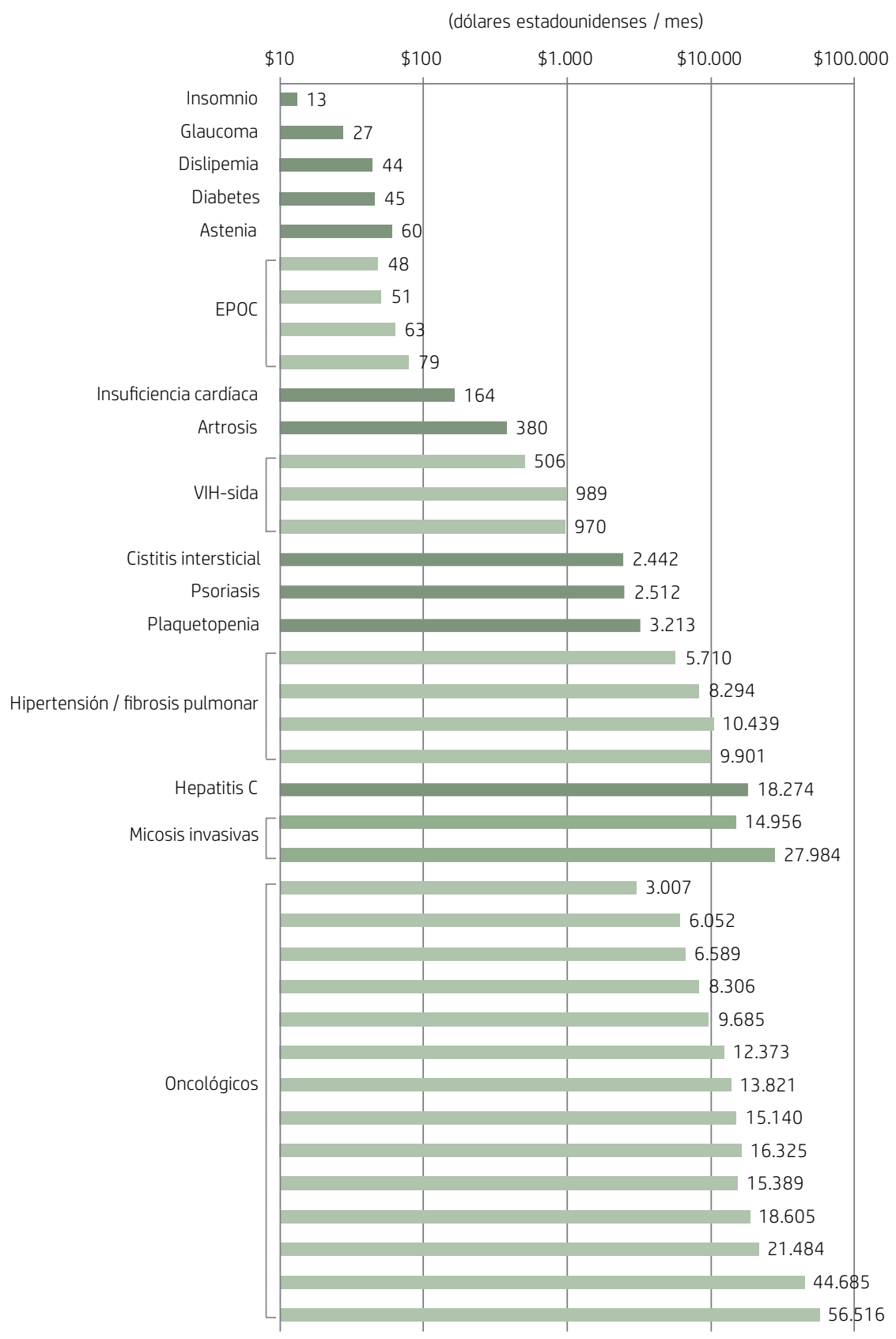

Figura 3. Nuevas especialidades medicinales aprobadas, según patología tratada y precio mensual del tratamiento en dólares estadounidenses. Argentina, 2016.

Fuente: Elaboración propia a partir de precios publicados en la revista Kairos ${ }^{(16)}$.

Nota: La escala de precios es logarítmica, a fin de facilitar la representación.

\section{DISCUSIÓN}

Este estudio encontró que, en el año 2016, se incorporaron al mercado argentino 45 NEM, la mayoría aprobadas previamente por la FDA y la EMA. El 13\% de ellas no tenían ensayos clínicos aleatorizados que demostraran eficacia y un $24 \%$ habían demostrado eficacia solo para variables subrogadas no validadas. En cuanto a su valor terapéutico, un $72 \%$ de las NEM no representaban avances según la evaluación de la revista Prescrire y un $78 \%$ no agregaban valor terapéutico según la 
clasificación de Ahlqvist-Rastad et al. En los dos casos evaluados solo por la agencia nacional, los criterios de aprobación utilizados no fueron robustos. Por otra parte, el precio de los nuevos productos fue sustancial, con una mediana de $\$ 5.849$ dólares mensuales para los fármacos de uso crónico. En definitiva, solo uno de cada cuatro NEM representa un avance terapéutico y casi todas salen a la venta con precios prácticamente inaccesibles.

Estos resultados concuerdan con los obtenidos en los estudios previamente citados. Las investigaciones realizadas en diferentes países de la región y del mundo coinciden en señalar la ausencia de valor terapéutico agregado de la mayor parte de los nuevos medicamentos comercializados. En síntesis, en el mejor de los casos, solo una cuarta parte de ellos representa algún tipo de mejora en las opciones terapéuticas disponibles.

El valor de los nuevos fármacos puede analizarse desde dos enfoques complementarios pero diferentes. El primero, contempla la innovación farmacéutica y valora, por ejemplo, la novedad de la estructura química, la diana molecular, el mecanismo de acción, la clase farmacológica, el método de síntesis o la formulación del medicamento ${ }^{(11,12)}$. El segundo enfoque privilegia el grado en que el nuevo fármaco representa un valor terapéutico agregado a las opciones previamente disponibles, es decir, refleja un beneficio adicional concreto para los pacientes ${ }^{(9,10)}$. Con el primer enfoque, que es el que aplican las agencias reguladoras, se puede considerar innovador a un fármaco nuevo que sea más eficaz que el placebo, mientras el segundo criterio, más exigente y con mayor relevancia sanitaria, requiere demostrar beneficio en ensayos clínicos sobre la mejor alternativa disponible.

Diversos estudios han comparado los nuevos agentes terapéuticos registrados por FDA, EMA y otras agencias reguladoras. Aunque no se encuentran diferencias significativas en los nuevos medicamentos, un estudio reciente observó que las indicaciones aprobadas por FDA, EMA y la agencia suiza Swissmedic (SMC) para los mismos fármacos diferían en contenido en el 76,9\% de los ca$\operatorname{sos}^{(33)}$. Está claro que las discrepancias entre las agencias reguladoras no solo se basan en la evidencia, sino que también influyen factores culturales, políticos y económicos y las características de los respectivos sistemas de atención médica ${ }^{(34)}$.

Aunque la asignación por la FDA de diferentes categorías como "revisión prioritaria" o "medicamento huérfano" pareciera promover el criterio de innovación más exigente, en la práctica son tantos los fármacos calificados de prioritarios que el término pierde especificidad y se vuelve indiscriminado. En cuanto a las enfermedades huérfanas investigadas, se trata por lo general de trastornos genéticos o de variantes oncológicas definidas por su muy baja prevalencia en EEUU, y no de las enfermedades infecciosas con alta prevalencia en el mundo en vías de desarrollo, para las que no existen todavía alternativas terapéuticas eficaces.

El valor terapéutico de una NEM es aún más incierto cuando, a través de las vías de "aprobación acelerada" que han establecido las agencias reguladoras, el fármaco sale al mercado con demostración de eficacia solo sobre variables subrogadas no validadas $\mathrm{o}$, incluso, sin haber realizado estudios controlados. Ejemplos de estas variables subrogadas cuestionadas son la caminata de seis minutos para la hipertensión arterial pulmonar ${ }^{(32)}$ y muchos de los desenlaces habituales en los estudios de fármacos oncológicos ${ }^{(35)}$. Los estudios poscomercialización, que requieren las agencias reguladoras para completar esta información, se demoran más de lo estipulado y a menudo no aportan la esperada información sobre desenlaces clínicamente relevantes $(24,25,36,37,38,39,40)$.

El resultado de esta modalidad más laxa de aprobación de fármacos es la incorporación de productos cuya eficacia y seguridad en variables clínicamente relevantes no está demostrada, con datos de seguridad preliminares y, por lo general, a un precio que compromete la viabilidad de los mecanismos de financiación.

La normativa argentina, que concede el registro a todo fármaco aprobado por las agencias reguladoras de los países desarrollados $^{(8)}$, reproduce localmente estos mismos 
problemas. Existen proyectos de ley para crear una agencia de evaluación de tecnologías sanitarias, que podría intervenir en la incorporación de un nuevo fármaco registrado a la cobertura de la seguridad social. Es imprescindible que para este tipo de decisiones se utilicen estándares que incorporen el valor terapéutico agregado por el nuevo fármaco, junto con el análisis de costo-efectividad frente a las alternativas.

Sin embargo, los resultados de los estudios analizados sugieren que para responder a las prioridades sanitarias locales se requieren cambios mayores en la normativa, abandonando el registro automático de lo aprobado por las agencias reguladoras de otros países. La nueva modalidad de registro debería incorporar la evaluación autónoma de los nuevos fármacos y aprobar el ingreso al mercado local de aquellos que representen verdaderos avances.

Como ejemplo de intervenciones de este tipo en países de la región, un decreto reciente de Colombia establece que el registro de nuevos medicamentos estará condicionado a la evaluación de su valor terapéutico con relación a un comparador seleccionado, junto con una evaluación económica, que podrá incluir un análisis de costo/efectividad y de impacto presupuestario(41). De este modo la evaluación del valor terapéutico y el establecimiento del precio son previos a la entrada al mercado del nuevo fármaco.

\section{CONCLUSIONES}

Como reflejo de lo que ocurre en los países desarrollados, solo una fracción minoritaria de los nuevos medicamentos aprobados en Argentina, en el año 2016, representa un avance terapéutico significativo. A pesar de ello, la mayoría de ellos tienen precios muy elevados. El resultado es un aumento de riesgos para los pacientes que se exponen a fármacos sin eficacia clínica comprobada y una sobrecarga para los sistemas de financiación públicos y privados. Para mejorar estos resultados se debería incorporar una evaluación del valor terapéutico y del precio del fármaco como requisito para autorizar el registro en el país de nuevos medicamentos o, como mínimo, realizar esta evaluación como paso previo a su aceptación para la cobertura por la seguridad social.

\section{AGRADECIMIENTOS}

Agradecemos a los editores de la revista Prescrire por facilitarnos el libre acceso a los artículos.

\section{REFERENCIAS BIBLIOGRÁFICAS}

1. Morgan SG, Bassett KL, Wright JM, Evans RG, Barer ML, Caetano PA, Black CD. "Breakthrough" drugs and growth in expenditure on prescription drugs in Canada. British Medical Journal. 2005;331 (7520):815-816.

2. National Institute for Health Care Management. Changing Patterns of Pharmaceutical Innovation [Internet]. Washington DC: The National Institute for Health Care Management Research and Educational Foundation; 2002 [citado 12 dic 2017]. Disponible en: https://tinyurl.com/yd3rc2q8.
3. Botelho SF, Martins MAP, Reis AMM. Analysis of new drugs registered in Brazil in view of the Unified Health System and the disease burden. Ciência \& Saúde Coletiva. 2018;23(1):215-228.

4. Editorial Staff. New products and new indications in 2016: a system that favours imitation over the pursuit of real progress. Prescrire International. 2017;26(182):136-139.

5. Ward DJ, Slade A, Genus T, Martino OI, Stevens AJ. How innovative are new drugs launched in the UK?: A retrospective study of new drugs listed in the British National Formulary (BNF) 2001-2012. BMJ Open. 2014;4(10):e006235. doi: 10.1136/ bmjopen-2014-006235.

6. Vitry $\mathrm{Al}$, Shin $\mathrm{NH}$, Vitre P. Assessment of the therapeutic value of new medicines marketed in Australia. Journal of Pharmaceutical Policy and Practice. 2013;6:2. doi: 10.1186/2052-3211-6-2. 
7. van Luijn JCF, Gribnau FWJ, Leufkens HGM. Superior efficacy of new medicines? European Journal of Clinical Pharmacology. 2010;66(5):445448.

8. Argentina, Poder Ejecutivo Nacional. Decreto 150/92: Normas para el registro, elaboración, fraccionamiento, prescripción, expendio, comercialización, exportación e importación de medicamentos; Ambito de aplicación; Disposiciones Generales [Internet]. 1992 [citado 1 mar 2018]. Disponible en: https://tinyurl.com/y79tdvq8.

9. Ahlqvist-Rastad J, Bardelay D, Beermann B, Mignot $G$. Judging the therapeutic value of drugs: A comparison between La revue Prescrire and Information från Läkemedelsverket, the bulletin of the Swedish Medical Products Agency. The International Journal of Risk \& Safety in Medicine. 2004;16(2):83-90.

10. Prescrire. Prescrire's ratings system: new drugs and indications, at a glance [Internet]. c2018 [citado 6 mar 2018]. Disponible en: https://tinyurl. com/ydx9muqn.

11. Aronson JK, Ferner RE, Hughes DA. Defining rewardable innovation in drug therapy. Nature Reviews Drug Discovery. 2012;11(4):253-254.

12. Motola D, De Ponti F, Poluzzi E, Martini N, Rossi P, Silvani MC, Vaccheri A, Montanaro N. An update on the first decade of the European centralized procedure: how many innovative drugs? British Journal of Clinical Pharmacology. 2006;62(5):610-616.

13. Homedes N, Ugalde A. Ensayos clínicos en América Latina: implicancias para la sustentabilidad y seguridad de los mercados farmacéuticos y el bienestar de los sujetos. Salud Colectiva. 2016;12(3):317-345.

14. Administración Nacional de Medicamentos, Alimentos y Tecnología Médica. Altas en el vademécum nacional de medicamentos [Internet]. c2018 [citado 1 mar 2018]. Disponible en: https:// tinyurl.com/y8rb5wog.

15. WHO Collaborating Centre for Drug Statistics Methodology. ATC/DDD Index 2018 [Internet]. c2017 [citado 1 mar 2018]. Disponible en: https:// tinyurl.com/yckuy4rt.

16. Kairos. 2016;XXXVIII(448):1-210.

17. Drugs@FDA: FDA Approved Drug Products [Internet]. c2018 [citado 1 mar 2018]. Disponible en: https://tinyurl.com//5mfqlh.

18. Center for Drug Evaluation and Research. Manual of policies and procedures [Internet].
2013 [citado 1 mar 2018]. Disponible en: https:// tinyurl.com/yamw8h6s.

19. European Medicines Agency [Internet]. c19952018 [citado 1 mar 2018]. Disponible en: https:// www.ema.europa.eu/.

20. Laporte JR, Porta M, Capella D. Drug utilization studies: a tool for determining the effectiveness of drug use. British Journal of Clinical Pharmacology. 1983;16(3):301-304.

21. United States, Food and Drug Administration. New drug, antibiotic, and biological drug product regulations: accelerated approval; Final rule. Federal Register. 1992;57(239):58942-58960.

22. FDA-NIH Biomarker Working Group. BEST (Biomarkers, EndpointS, and other Tools) Resource [Internet]. 2016 [citado 5 mar 2018]. Disponible en: https://tinyurl.com/ydbcc2er.

23. Twaddell S. Surrogate outcome markers in research and clinical practice. Australian Prescriber. 2009;32(2):47-50.

24. Kim C, Prasad V. Cancer drugs approved on the basis of a surrogate end point and subsequent overall survival: an analysis of 5 years of US food and drug administration approvals. JAMA Internal Medicine. 2015;175(12):1992-1994.

25. Rupp T, Zuckerman D. Quality of life, overall survival, and costs of cancer drugs approved based on surrogate endpoints. JAMA Internal Medicine. 2017; 177(2):276-277.

26. Administración Nacional de Medicamentos, Alimentos y Tecnología Médica. Disposición 8806/16 [Internet]. 2016 [citado 1 mar 2018]. Disponible en: https://tinyurl.com/y7gfs6kv.

27. Administración Nacional de Medicamentos, Alimentos y Tecnología Médica. Disposición 0759/16 [Internet]. 2016 [citado 1 mar 2018]. Disponible en: https://tinyurl.com/y7u7ejug.

28. Esteban C, Quintana JM, Aburto M, Moraza J, Egurrola M, España PP, Pérez-Izquierdo J, Capelastegui A. Predictors of mortality in patients with stable COPD. Journal of General Internal Medicine. 2008;23(11):1829-1834.

29. Traver GA, Cline MG, Burrows B. Predictors of mortality in chronic obstructive pulmonary disease: A 15-year follow-up study. The American Review Of Respiratory Disease. 1979;119(6):895-902.

30. Mishra P, Murray J, Birnkrant D. Direct-acting antiviral drug approvals for treatment of chronic hepatitis $C$ virus infection: Scientific and regulatory 
approaches to clinical trial designs. Hepatology: Official Journal of the American Association for the Study of Liver Diseases. 2015;62(4):12981303.

31. van der Meer AJ, Veldt BJ, Feld JJ, Wedemeyer $\mathrm{H}$, Dufour J-F, Lammert F, Duarte-Rojo A, Heathcote EJ, et al. Association between sustained virological response and all-cause mortality among patients with chronic hepatitis $C$ and advanced hepatic fibrosis. JAMA. 2012;308(24):2584-2593.

32. Gabler NB, French B, Strom BL, Palevsky HI, Taichman DB, Kawut SM, Halpern SD. Validation of 6-minute walk distance as a surrogate end point in pulmonary arterial hypertension trials. Circulation. 2012;126(3):349-356.

33. Zeukeng MJ, Seoane-Vazquez E, Bonnabry P. A comparison of new drugs approved by the FDA, the EMA, and Swissmedic: an assessment of the international harmonization of drugs. European Journal of Clinical Pharmacology. 2018;74(6):811-818.

34. Braillon A. The race for drug approvals: hasten slowly? European Journal of Clinical Pharmacology. 2018;74(9):1197-1198.

35. Prasad V, Kim C, Burotto M, Vandross A. The strength of association between surrogate end points and survival in oncology: A systematic review of trial-level meta-analyses. JAMA Internal Medicine. 2015;175(8):1389-1398.
36. Pease AM, Krumholz HM, Downing NS, Aminawung JA, Shah ND, Ross JS. Postapproval studies of drugs initially approved by the FDA on the basis of limited evidence: systematic review. BMJ: British Medical Journal. 2017;357:j1680. doi: 10.1136/bmj.j1680.

37. Naci H, Smalley KR, Kesselheim AS. Characteristics of preapproval and postapproval studies for drugs granted accelerated approval by the Us Food and Drug Administration. JAMA. 2017;318(7):626-636.

38. Knopf K, Baum M, Shimp WS, Bennett CL, Faith D, Fishman ML, Hrushesky WJ. Interpretation of surrogate endpoints in the era of the $21 \mathrm{st}$ Century Cures Act. BMJ: British Medical Journal. 2016;355:i6286. doi: 10.1136/bmj.i6286.

39. Gellad WF, Kesselheim AS. Accelerated approval and expensive drugs: A challenging combination. The New England Journal of Medicine. 2017;376(21):2001-2004.

40. Hawkes N. German body calls for pause in European plan for fast track drug approval. BMJ. 2016;354:i4479. doi: https://doi.org/10.1136/bmj. i4479.

41. Colombia, Ministerio de Salud. Decreto 433 de 2018 [Internet]. 2018 [citado 15 mar 2018]. Disponible en: https://tinyurl.com/y78xoxz2. 